\title{
SYSTEMATIC REVIEW OF THE USE OF NONINVASIVE NEURALLY ADJUSTED VENTILATORY ASSIST VS. CONVENTIONAL NONINVASIVE VENTILATION IN PEDIATRIC PATIENTS WITH ACUTE RESPIRATORY FAILURE
}

\author{
João Souza ${ }^{1}$, Celso Rebello ${ }^{1}$, Carlos Oliveira ${ }^{1}$, and Eduardo Troster ${ }^{1}$ \\ ${ }^{1}$ Hospital Israelita Albert Einstein
}

October 27, 2020

\begin{abstract}
The use of noninvasive ventilation (NIV) is considered for the treatment of mild and moderate cases of acute respiratory failure (ARF). Conventional trigger modes during NIV have a higher rate of asynchronies when compared to the electrical activity of the diaphragm (AEdi) trigger. Based on previous studies, the hypothesis of the present study is that there is evidence of lower rate of asynchronies and favorable clinical outcomes related to the non-invasive neurally adjusted ventilatory assist (NIV NAVA) mode when compared to the use of conventional NIV in pediatric patients with ARF. Purpose: To compare the asynchrony index (AI) and clinical outcomes presented during the use of NIV NAVA vs. conventional NIV in the treatment of ARF in pediatrics. Methods: This is a systematic review of clinical trials conducted between April and May 2020 in the electronic databases Cochrane Library, Embase, Lilacs, Pubmed/Medline, Scopus and Web of Science. Results: A total of 184 studies were found, four of which were eligible for qualitative synthesis. The sample analyzed 39 participants, aged between 35 days and 15 years with male predominance $(61.5 \%)$. The primary outcome analyzed in three of the four studies was the significant decrease $(\mathrm{p}<0.001)$ in the AI during NIV NAVA compared to conventional NIV. Clinical outcomes were inconclusive due to methodological limitations. Conclusion: We conclude that NIV NAVA decreases the AI when compared to conventional NIV in pediatric patients with ARF. However, the other clinical outcomes present inconclusive results, requiring further studies with different methodological formats for confirmation.
\end{abstract}

SYSTEMATIC REVIEW OF THE USE OF NONINVASIVE NEURALLY ADJUSTED VENTILATORY ASSIST VS. CONVENTIONAL NONINVASIVE VENTILATION IN PEDIATRIC PATIENTS WITH ACUTE RESPIRATORY FAILURE

João Marcos Feliciano de Souza; Celso Moura Rebello; Carlos Augusto Cardim de Oliveira; Eduardo Juan Troster.

São Paulo

2020

\section{LIST OF ABBREVIATIONS}

AI Asynchrony Index 
ARF Acute Respiratory Failure

AT Automatic Trigger

AVB Acute Viral Bronchiolitis

DT Double Trigger

EAdi Electrical Activity of the diaphragm

ET Expiratory Time

FiO2 Fraction-inspired Oxygen

HR Heart Rate

ICU Intensive Care Unit

IE Ineffective Effort

IT Inspiratory Time

LC Latte Cycling

MeSH Medical Subject Headings

mmHg Millimeters of mercury

MV Mechanical Ventilation

NCPAP Nasal Continuous Positive Airway Pressure

NIPPV Nasal Intermittent Positive Pressure Ventilation

NIV NAVA Noninvasive Neurally Adjusted Ventilatory Assist

NIV Noninvasive Ventilation

OI Oxygenation Index

PAC Pressure Assist Control

PaCO2 Carbon dioxide blood Pressure

$\mathrm{PaO} 2$ Oxygen blood Pressure

PC Premature Cycling

PEEP Positive End-Expiratory Pressure

pH Hydrogenionic potential

PRISMA Preferred Reporting Items for Systematic reviews and Meta-Analyses

PS Pressure Support

RoB Risk of Bias

RR Respiratory Rate

SNIPPV Synchronized Nasal Intermittent Positive Pressure Ventilation

SpO2 Peripheral capillary oxygen Saturation

TcCO2 Transcutaneous carbon dioxide

VILI Ventilation Induced Lung Injury 
Vmin Volume minute

VS. Versus

\begin{abstract}
Introduction: The use of noninvasive ventilation (NIV) is considered for the treatment of mild and moderate cases of acute respiratory failure (ARF). Conventional trigger modes during NIV have a higher rate of asynchronies when compared to the electrical activity of the diaphragm (AEdi) trigger. Based on previous studies, the hypothesis of the present study is that there is evidence of lower rate of asynchronies and favorable clinical outcomes related to the non-invasive neurally adjusted ventilatory assist (NIV NAVA) mode when compared to the use of conventional NIV in pediatric patients with ARF.Purpose: To compare the asynchrony index (AI) and clinical outcomes presented during the use of NIV NAVA vs. conventional NIV in the treatment of ARF in pediatrics. Methods: This is a systematic review of clinical trials conducted between April and May 2020 in the electronic databases Cochrane Library, Embase, Lilacs, Pubmed/Medline, Scopus and Web of Science. Results: A total of 184 studies were found, four of which were eligible for qualitative synthesis. The sample analyzed 39 participants, aged between 35 days and 15 years with male predominance $(61.5 \%)$. The primary outcome analyzed in three of the four studies was the significant decrease $(\mathrm{p}<0.001)$ in the AI during NIV NAVA compared to conventional NIV. Clinical outcomes were inconclusive due to methodological limitations. Conclusion: We conclude that NIV NAVA decreases the AI when compared to conventional NIV in pediatric patients with ARF. However, the other clinical outcomes present inconclusive results, requiring further studies with different methodological formats for confirmation.
\end{abstract}

PROSPERO ID: 181785

Keywords: Noninvasive Ventilation, Child, Support, Interactive Ventilatory, Neurally Adjusted Ventilatory Assist.

\title{
1 INTRODUCTION
}

The Acute Respiratory Failure (ARF) is the most prevalent comorbidity in children hospitalized in the Intensive Care Unit (ICU) worldwide. ${ }^{(1)}$ According to the classification of severity and impairment of the respiratory system, Noninvasive Ventilation (NIV) will be indicated as the primary choice in the treatment of $\mathrm{ARF}$. $(2)$

Conventional NIV has pneumatic components that, when detecting changes in flow or pressure, synchronize the patient's respiratory efforts to the inspiratory flows delivered. Thus, the choice of interfaces with lower leakage rates is directly related to better synchrony between patient and mechanical ventilator. ${ }^{(3)}$

During Noninvasive Neurally Adjusted Ventilatory Assist (NIV NAVA) mode, transesophageal electrodes sensitive to changes in Electrical Activity of diaphragm (EAdi) exclude the pneumatic component and synchronize inspiratory flow proportionally to respiratory efforts. That is, the greater the diaphragmatic excitation of the patient, the higher the peak of positive inspiratory pressure delivered. ${ }^{(4)}$

The interaction between the patient and the mechanical ventilator is evaluated by means of the Asynchrony Index (AI), obtained by the sum of asynchronous events: Ineffective Efforts (IE), Automatic Trigger (AT), Double Trigger (DT), Premature Cycling (PC) and Late Cycling (LC), divided by the sum of respiratory cycles and IE multiplied by 100 . The calculation: $\mathrm{AI}=[(\mathrm{IE}+\mathrm{AT}+\mathrm{DT}+\mathrm{PC}+\mathrm{LC}) \div($ respiratory cycles $+\mathrm{IE})] \times 100$, will result in a percentage of asynchrony. ${ }^{(5,6)}$ Rates greater than or equal to $10 \%$ are associated with unfavorable clinical outcomes, such as: changes in tidal volume delivery, increased respiratory work, increased need for the use of sedatives, increased time on mechanical ventilation (MV), increased length of stay in the ICU, in addition to higher mortality rates and Ventilation Induced Lung Injury (VILI). ${ }^{(7,8)}$ 
Due to what was mentioned, the present study presents two possible hypotheses. The first is that there is correlated evidence between the use of NIV NAVA and lower AI when compared to Conventional NIV in pediatric patients with ARF. The second is that there is correlated evidence between the use of NIV NAVA and favorable clinical outcomes when compared to Conventional NIV in pediatric patients with ARF.

Thus, the aim of this systematic review is to analyze and compare the asynchronous events and clinical outcomes of patients during the use of NIV NAVA vs. Conventional NIV in the treatment of ARF in children aged between 1 month and 18 years.

\section{Objective}

Compare AI during the use of NIV NAVA vs. Conventional NIV in the treatment of ARF in pediatric patients aged 1 month to 18 years.

To compare clinical outcomes during the use of NIV NAVA vs. Conventional NIV in the treatment of AI in pediatric patients aged 1 month to 18 years.

\section{METHODS}

The Preferred Reporting Items for Systematic reviews and Meta-Analyses (PRISMA) guidelines were used to delimit the processes of this review. Clinical trials were selected, comparing the use of NIV NAVA vs. Conventional NIV in the treatment of ARF in pediatric patients, aged between 1 month and 18 years of age incomplete. The study protocol was registered in PROSPERO: International Prospective Register of Systematic Reviews (ID: 181785).

\subsection{Search strategy}

The searches were performed by two independent reviewers in the electronic databases of the Cochrane Library, Embase, Lilacs, Pubmed/Medline, Scopus and Web of Science between April and May 2020.

Using the thematic blocks: Noninvasive Ventilation, Children and Neurally Adjusted Ventilatory Assist, the research combined the terms of Medical Subject Headings (MeSH) with Boolean operators "OR" and "AND" as follows: 'Noninvasive Ventilations' OR 'Ventilation, Noninvasive' OR 'Ventilations, Noninvasive' OR 'Non-Invasive Ventilation' OR 'Non-Invasive Ventilations' OR 'Ventilation, Non-Invasive' OR 'Ventilations, Non-Invasive' OR 'Non Invasive Ventilation' OR 'Non Invasive Ventilations' OR 'Ventilation, Non Invasive' OR 'Ventilations, Non Invasive'; AND 'Children' OR 'Preschool Child' OR 'Children, Preschool' OR 'Preschool Children Adolescent' OR 'Child'; AND 'Support, Interactive Ventilatory' OR 'Ventilatory Support, Interactive' OR 'Neurally Adjusted Ventilatory Assist'.

\subsection{Inclusion criteria}

The primary analysis of the articles was made after reading the title and abstract, applying the following criteria for inclusion: 1. Randomized clinical trials; 2. Age group of research subjects between 1 month and 18 years incomplete; 3 . Patients with respiratory failure; 4. In use of the following NIV modes: (a) Nasal Intermittent Positive Pressure Ventilation (NIPPV), (b) Synchronized Nasal Intermittent Positive Pressure Ventilation (SNIPPV) or NIV NAVA.

\subsection{Exclusion criteria}

With no restrictions on the type of language and year of publication, the following exclusion criteria were applied: 1. Retrospective and prospective observational studies; 2. Studies on non-human experimental models; 2. Publications without the full results available for individual analysis of the research subjects; 3 . Studies in 
populations composed exclusively of newborns or adults; 4. Patients using NIV by Continuous Nasal Airway Pressure (NCPAP) mode; 7. Patients using invasive MV and 8. Interrupted or ongoing studies.

\subsection{Risk of bias analysis}

Through the Risk of Bias (RoB) tool of the Cochrane Library, it was possible to evaluate the methodological quality and internal validity of the studies. Classifying them as high, low or uncertain risk of bias, the following criteria were applied: 1 . Generation of the randomization sequence; 2. Confidentiality of allocation; 3. Concealment of allocation; 4. Masking (blinding) of participants and the team of researchers; 5 . Masking (blinding) in the evaluation of outcomes; 6 . Incomplete data of outcomes; 7. Selective report of outcomes and 8 . Other sources of bias identified by the reviewer. ${ }^{(9)}$

\section{RESULTS}

\subsection{Search results}

The bibliographic research conducted between April and May 2020 resulted in the identification of 184 articles. There were 69 obtained from the Cochrane Library's electronic database, 41 from Embase, 22 from Lilacs, 22 from Pubmed/Medline, five from Scopus and 25 from Web of Science.

After the removal of 58 duplicate articles, another 122 were excluded because they did not meet the inclusion criteria during the analysis of the title and abstract as observed in Figure 1.

The selection process included four articles for qualitative analysis (Table 1). Two randomized clinical trials $(10,11)$ and two non-randomized clinical trials. ${ }^{(12,13)}$

\subsection{Sample size}

The sample size of the four articles ranged from six to 18 participants ${ }^{(10,11)}$, resulting in 48 participants in total.

During the individual analysis of the research subjects, nine (18.75\%) were excluded from the analyses because they did not meet the inclusion criteria of age group (less than 1 month) and ventilatory mode (NCPAP). Thus, the total of $39(81.25 \%)$ subjects were effectively included in the qualitative analysis (Table $1)$. 


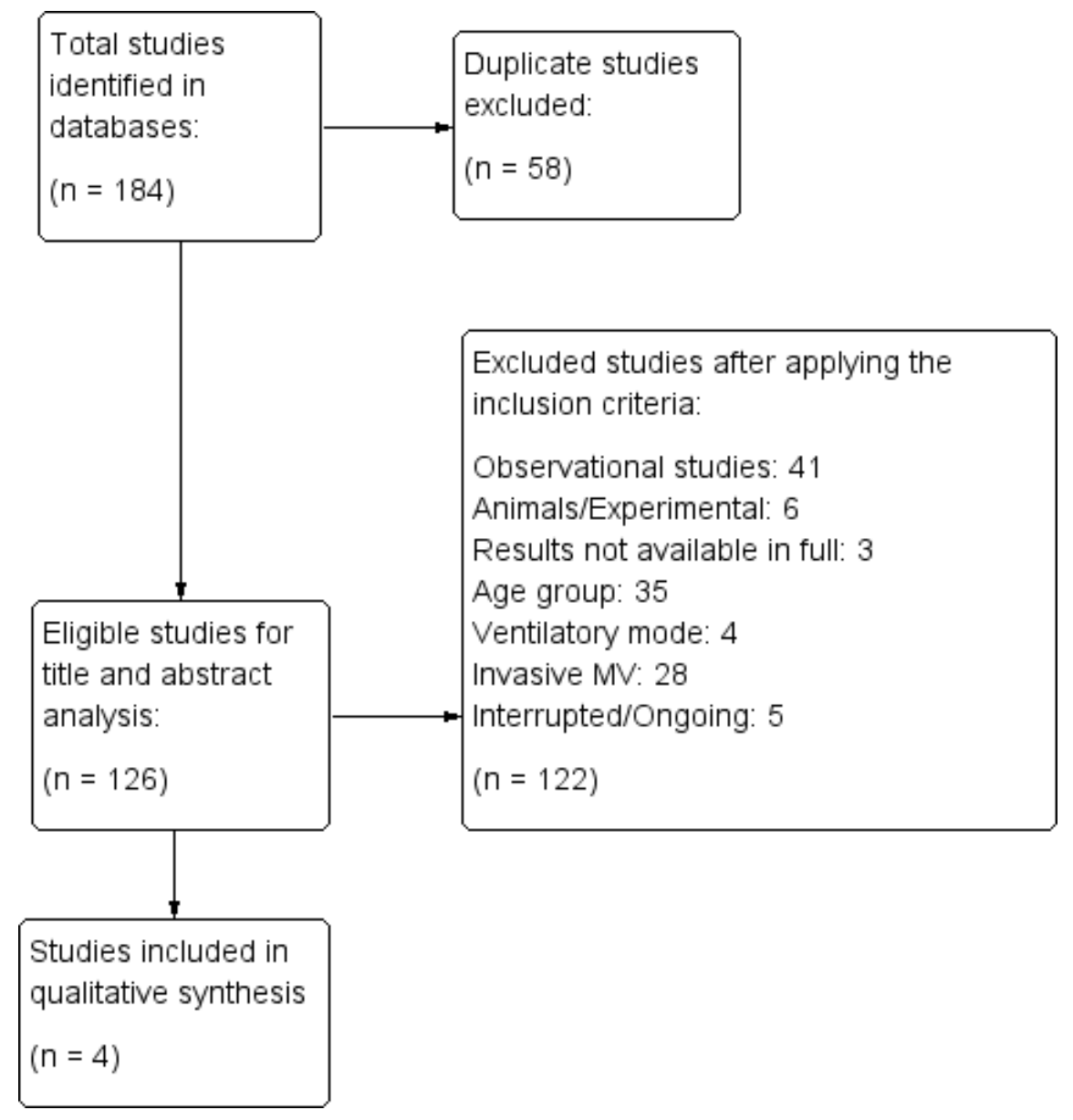

Figure 1. Research flow diagram

\subsection{Age and gender}

The age of the eligible subjects ranged from 35 days of life to 15 years and 10 months, with a male predominance ranging from $66 \%$ to $80 \%$ (Table 1). The analysis of the study by Vignaux et al. 2013, presented a median age of 18 months of the total sample $(n=6)$, with interquartile interval (IIQ) $1=5$ - IIQ3 $=27$. In addition to male predominance in $66 \%{ }^{(10)}$

In the study by Baudin et al. 2014, the median age of the total sample ( $\mathrm{n}=11)$ was 0.9 months $(0.53-1.7)$. However, six subjects (54\%) were excluded from the analysis because they did not meet the inclusion criteria by age group. Thus, the median age of the eligible sample $(\mathrm{n}=5)$ becomes 1.87 months $(1.53-2.1)$, with a male predominance of $80 \%{ }^{(12)}$

In the study by Ducharme et al. 2015, the median age of the total sample $(\mathrm{n}=13)$ was 42 months $(2$ $76)$. However, three subjects $(23 \%)$ were excluded from the analysis because they did not meet the inclusion criteria in a ventilatory mode (NCPAP). Thus, the median age of the eligible sample $(\mathrm{n}=10)$ becomes 53.75 months $(16.5-125.5)$, with a male predominance of $70 \% .^{(13)}$

In the study by Chidini et al. 2016, the median age of the total sample $(\mathrm{n}=18)$ was 13 months $(8.5-17.75)$, with no gender predominance. ${ }^{(11)}$ 


\begin{tabular}{lllll}
\hline Author/Year & Intervention sequence & Total sample $(\mathrm{n}=48)$ & Eligible sample $(\mathrm{n}=39)$ & Median age $($ months $) c$ \\
\hline Vignaux et al. 2013 & Randomized & 6 & 6 & $18(5-27)$ \\
Baudin et al. 2014 & Non-randomized & 11 & 5 & $1,87(1,53-2,1)$ \\
Ducharme et al. 2015 & Non-randomized & 13 & 10 & $53,75(16,5-125,5)$ \\
Chidini et al. 2016 & Randomized & 18 & 18 & $13(8,5-17,75)$ \\
\hline
\end{tabular}

Table 1. Sample characteristics

\subsection{Classification}

All studies classified the subjects based on the AI during the period of hospitalization in the pediatric ICU.

The relationship between oxygen blood pressure and fraction-inspired oxygen (PaO2/FiO2) less than 300, respiratory rate $(\mathrm{RR})$ above the predicted value for age, use of accessory respiratory muscles and difficulty in feeding were the classification criteria used by Chidini et al. 2016. ${ }^{(11)}$

The continuous use of NIV for a period longer than 6 hours was one of the classification criteria in the study by Ducharme et al. 2015. ${ }^{(13)}$ Therapeutic failure in the use of NCPAP, recurrent apnea events in an interval of less than 1 hour, signs of respiratory distress with gasometric changes such as: hydrogenonic potential $(\mathrm{pH})$ less than 7.30 and carbon dioxide blood pressure $(\mathrm{PaCO} 2)$ greater than 60 millimeters of mercury $(\mathrm{mmHg})$ were the classification criteria adopted by Baudin et al. 2014. ${ }^{(12)}$ While Vignaux et al. 2013 applied NIV after extubation of intubated patients for surgical procedures. ${ }^{(10)}$

\subsection{Intervention}

According to the cross-over method adopted by the authors, all subjects participated simultaneously in the NIV NAVA and Conventional NIV group (Table 2).

The period of analysis of the outcomes in each group ranged from 10 to 60 minutes. Since the time interval between interventions, called "washout period", ranged from five to 70 minutes in the studies by Vignaux et al. 2013, Baudin et al. 2014 and Chidini et al. 2016.(10-12) The washout period was not described in the studies by Ducharme et al. 2015. ${ }^{(13)}$

During NIV NAVA and Conventional NIV, only one device (Servo I \& - Maquet) was used in Vignaux et al. 2013, Baudin et al. 2014 and Chidini et al. 2016 studies. ${ }^{(10-12)}$ During Ducharme et al. studies, NIV NAVA was used by Servo i ( - Maquet, but during Conventional NIV five different devices (Babylog @ 8000 plus - Draeger; Servo i @ - Maquet; VPAP III @ - ResMed; BIPAP $₫$ and Trilogy 100 ( - Philips) was described. ${ }^{(13)}$ Five different types of NIV interfaces have also been described. Face mask and nasal prong were used by Vignaux et al. $2013^{(10)}$, nasal mask was used by Baudin et al. $2014^{(12)}$, nasopharyngeal tubes, nasal masks and nasobuccal (face mask) were used by Ducarme et al. $2015^{(13)}$, and only total face mask was used by Chidini et al. 2016. ${ }^{(11)}$

Vignaux et al. 2013 applied randomly, a total of 25 minutes of Conventional NIV (Pressure Support - PS)

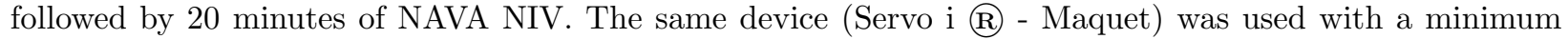
washout period of five minutes. While the choice of interfaces (facial mask and nasal prong) could vary according to the tolerance and agitation of the participant. ${ }^{(10)}$

Baudin et al. 2014 applied a non-randomized sequence with two hours in Conventional NIV (Pressure Assist Control - PAC) followed by a washout period between 110 and 130 minutes, and finally another 2 hours in NIV NAVA. The authors used only the last 10 minutes of each intervention for comparative analyses. The same device (Servo I B - Maquet) and interface (nasal mask) were used in both interventions. ${ }^{(12)}$ 
Ducharme et al. 2015 applied a non-randomized sequence, without a specific washout period, composed of three interventions periods. Initially 30 minutes in Conventional NIV (NCPAP, PAC or PS), followed by 60 minutes in NIV NAVA, and finally another 30 minutes in Conventional NIV. The authors used only the last 10 minutes of each intervention period for comparative analyses. Five different types of devices (Babylog 8000@ - Draeger; Servo i B. - Maquet; VPAP III \& - ResMed; BIPAP (B) and Trilogy 100 ( ) Philips) and interfaces (nasopharyngeal tubes, nasal masks and nasobuccal (face mask)) were used in this study. ${ }^{(13)}$

Chidini et al. 2016 applied randomly 60 minutes of Conventional NIV (PS) and 60 minutes of NIV NAVA,

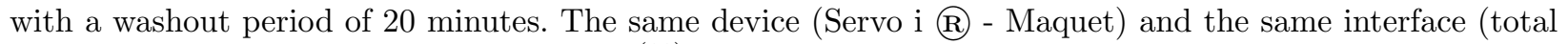
face mask) were used in both interventions. ${ }^{(11)}$

Table 2. Methodological data from selected studies

Author/Year

Total sample

( $\mathrm{n}=48)$

Device

Interface

NIV NAVA

period

(minutes)

Conventional NIV

period

(minutes)

Vignaux et al. 2013

6

Servo i \&

Face mask or Nasal prong

$>5$

20

25

Baudin et al. 2014

11

Servo i \&

Nasal mask

$110-130$

10

10

Ducharme et al. 2015 
Various

Various

There is no description

10

20

Chidini et al. 2016

18

Servo i ß

Total face mask

20

60

60

\subsection{Outcomes}

The primary outcome observed in three of the four articles was the AI (Table 3). It was possible to observe a significant decrease in AI during NIV NAVA when compared to Conventional NIV ( $\mathrm{p}<0.001) .{ }^{(10-12)}$

In the study by Ducharme et al. 2015 synchrony-related outcomes were quantified according to the percentage of time spend on asynchronies, and not by AI. It was possible to observe a decrease in the percentage of time spend on asynchronies during NIV NAVA when compared to Conventional NIV $(\mathrm{p}=0.05)$. The authors defined as primary outcome the feasibility and tolerance of NIV NAVA. Concluding that its use was feasible, according to its ability to obtain a correct signal from the AEdi, to decrease the percentage of time spend on asynchronies and by not interrupting the ventilatory support, besides being tolerable because it did not present differences in the clinical conditions of ICU patients. ${ }^{(13)}$

Other outcomes such as mean ventilatory parameters, clinical characteristics, gas exchange and sedative use were also quantified (Table 3). Presenting significant increase in the values of: inspiratory peak pressure (p $=0.03)$ and mean airway pressure $(\mathrm{p}=0.016)$ during NAVA NIV. There were no significant changes in the values of $R R(p=0.37)$, tidal volume $(\mathrm{p}=0.562)$, peak of EAdi $(\mathrm{p}=0.077)$, neural Inspiratory Time (IT) ( $\mathrm{p}$ $=0.95)$ and percentage of leaks $(\mathrm{p}=0.817)$ between interventions in the studies by Vignaux et al. 2013. ${ }^{(10)}$

The studies of Baudin et al. 2014, showed a significant increase in MV RR ( $\mathrm{p}=0.03)$ during NIV NAVA. There were no significant changes in the values of inspiratory peak pressure $(\mathrm{p}=0.09), \mathrm{FiO} 2(\mathrm{p}=0.22)$, Positive End-Expiratory Pressure (PEEP) $(p=0.95)$, neural RR $(p=0.16)$, peak EAdi $(p=0.80)$, peripheral capillary oxygen saturation $(\mathrm{SpO} 2)(\mathrm{p}=0.07)$, Heart Rate $(\mathrm{HR})(\mathrm{p}=0.18)$, transcutaneous carbon dioxide $(\mathrm{TcCO} 2)(\mathrm{p}=0.3)$ and respiratory distress $(\mathrm{p}=0.87)$ between interventions. $^{(12)}$

The studies of Chidini et al. 2016, presented significant changes in the decrease in oxygenation index (OI) (p $=0.043)$, peak inspiratory flow $(\mathrm{p}=0.001)$, inspiratory peak pressure $(\mathrm{p}=0.006)$ and mean airway pressure $(\mathrm{p}=0.038)$, in addition to increased minute volume $(\mathrm{Vmin})(\mathrm{p}=0.038)$ and neural $\mathrm{RR}(\mathrm{p}=0.013)$ during NIV NAVA. There were no significant changes in $\mathrm{pH}, \mathrm{PaCO} 2, \mathrm{PaO} 2 / \mathrm{FiO} 2$, use of sedatives $(\mathrm{p}=0.752)$, FiO2 $(\mathrm{p}=0.871)$, tidal volume $(\mathrm{p}=0.631)$, Neural IT $(\mathrm{p}=0.051)$, neural Expiratory Time $(\mathrm{ET})(\mathrm{p}=$ $0.657)$ and peak EAdi $(\mathrm{p}=0.013)$ between interventions. ${ }^{(11)}$

Table 3. Descriptive analysis of selected studies 


\begin{tabular}{lll}
\hline Author/Year & Outcomes & Description \\
\hline Vignaux et al. 2013 & AI & AI reduction during NIV NAVA $(\mathrm{p}<0.001)$. \\
& Average of ventilatory parameters & Increased inspiratory peak pressure $(\mathrm{p}=0.03)$ and me \\
Baudin et al. 2014 & AI & AI reduction during NIV NAVA $(\mathrm{p}<0.0001)$. \\
& Average of ventilatory parameters & Increased MV RR (p = 0.03) during NIV NAVA. Ther \\
& Clinical characteristics & There were no significant changes in SpO2, HR, TcCO \\
Ducharme et al. 2015 & NIV NAVA feasibility and tolerance & NIV NAVA is feasible and tolerable in critically ill chil \\
& Percentage of time spend on asynchronies & Decrease in the percentage of time spent on asynchron \\
Chidini et al. 2016 & AI & AI reduction during NIV NAVA (p $<0.001)$. \\
& Average of ventilatory parameters & Decrease in peak inspiratory flow (p =0.001), peak ins \\
& Gas exchange and use of sedatives & Decrease in OI $(\mathrm{p}=0.043)$ during NIV NAVA. There \\
\end{tabular}

\subsection{Risk of bias analysis}

The methodological quality analysis of the studies, observed in Figure 2 and Figure 3, was obtained using the evaluation criteria of the RoB tool. ${ }^{(9)}$ With the exception of the study published by Chidini et al. 2016, all classified at least one item as being "high risk" or "uncertain risk" of methodological bias.

The non-randomization of the interventions applied by Baudin et al. 2014 and Ducharme et al. 2015, present a "high risk" of methodological bias. ${ }^{(12,13)}$

The non-blinding in the allocation of subjects in the studies published by Baudin et al. 2014 and Ducharme et al. 2015 present a "high risk" of bias. ${ }^{(12,13)}$ While the non-description of this item in the study published by Vignaux et al. 2013, classifies it as an "uncertain risk" of bias. ${ }^{(10)}$

In none of the four articles analyzed, the subjects or the research team were blinded. According to the judgment of the authors of this review, this item did not present interferences in the analysis of the results. Thus, the studies are classified as "low risk" of bias. ${ }^{(10-13)}$

Blinding during the evaluation of outcomes was described in the studies by Baudin et al. 2014 and Chidini et al. 2016, and was classified as "low risk" of bias. ${ }^{(11,12)}$ The non-description of this item in the studies by Vignaux et al. 2013 and Ducharme et al. 2015 was classified as "uncertain risk" of bias. ${ }^{(10,13)}$

All studies appear to be free of friction and communication bias, related respectively to incomplete data and selective reporting of outcomes. ${ }^{(10-13)}$

Other risks such as the use of two types of interfaces, the heterogeneity of the subjects and the restricted analysis of breaths triggered by the EAdi, appear a "high risk" of bias in the analysis of the results published by Vignaux et al. 2013. ${ }^{(10)}$ As well as the non-description of washout period, the use of several types of interfaces and MV devices during the application of the interventions, classified the study by Ducharme et al. as "high risk" of bias. ${ }^{(13)}$ 


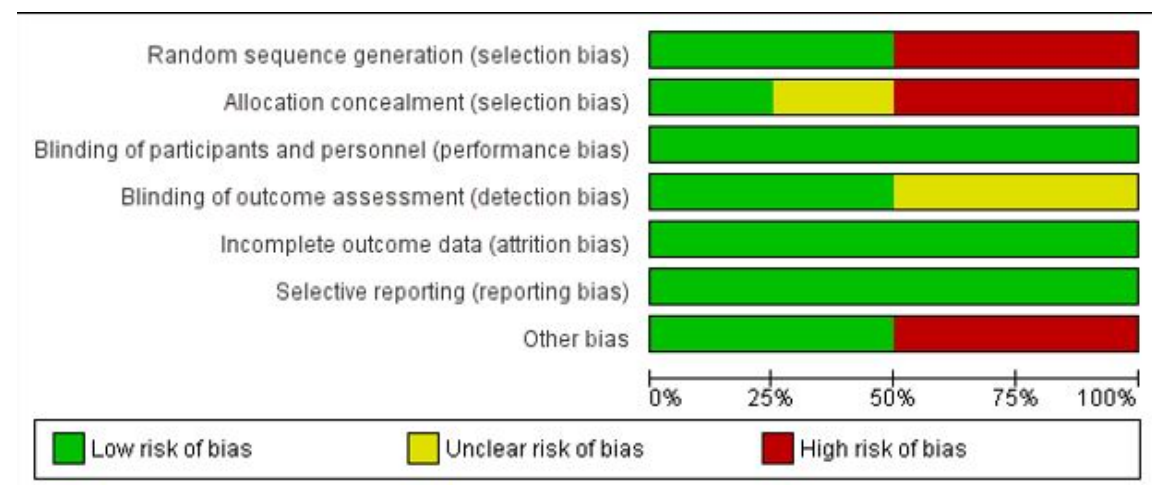

Figure 2. Risk of bias analysis graph: review the authors' judgments about each bias risk item presented as a percentage in all included studies. 


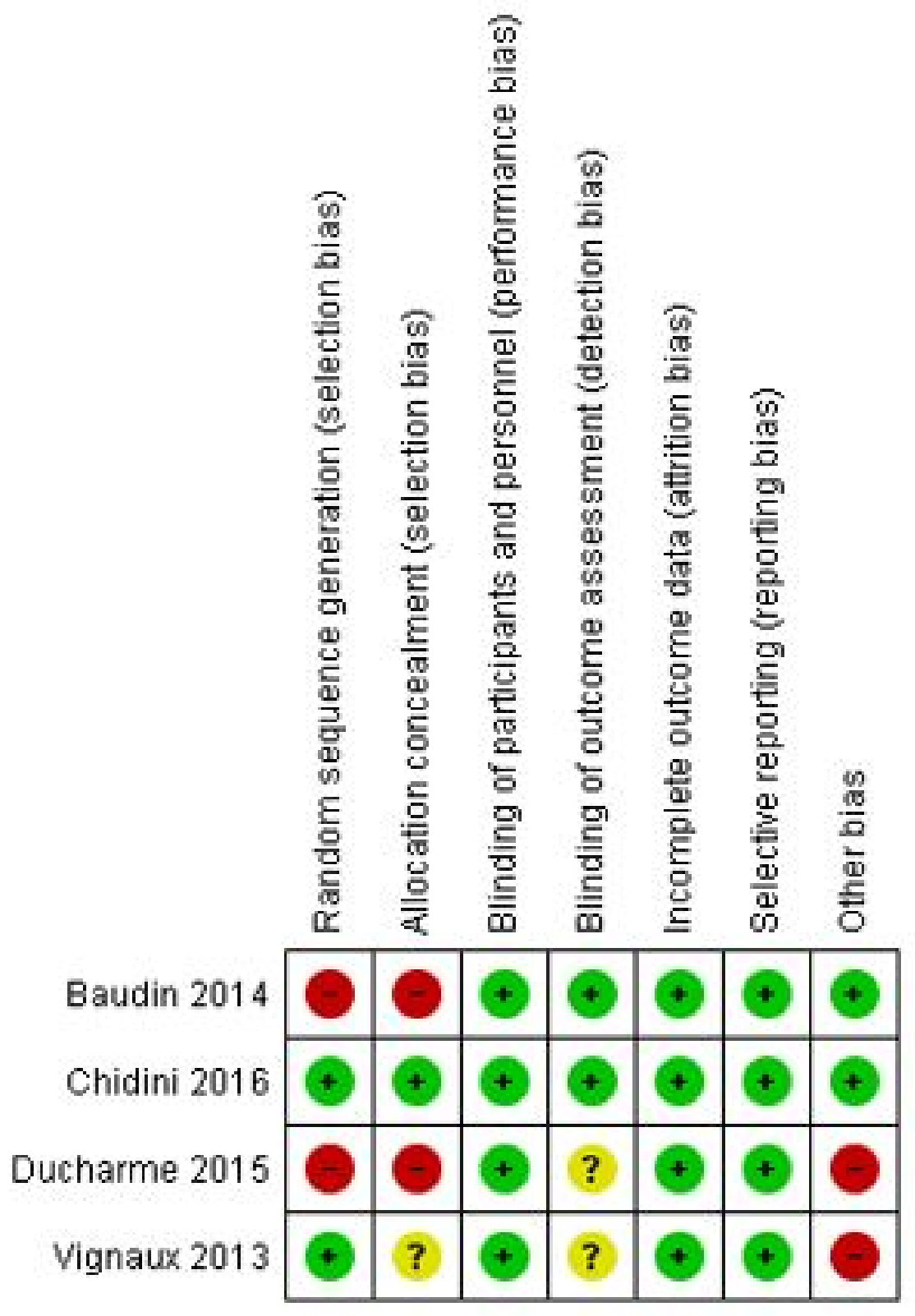

Figure 3. Summary of the risk of bias: analyze the authors' judgments about each risk item of bias for each study included.

\section{DISCUSSION}

Four studies that analyzed the synchrony and clinical outcomes associated with the application of NIV NAVA compared to Conventional NIV during the treatment of children of 39 children (eligible) with ARF. ${ }^{(10-13)}$

Three studies showed a significant decrease $(\mathrm{p}<0.001)$ in AI during NIV NAVA ${ }^{(10-12)}$, while one study showed a decrease in the percentage of time spent on asynchronies during NIV NAVA $(\mathrm{p}=0.05) .{ }^{(13)}$ According to the authors, the choice of this type of variable was due to the particular importance of inspiratory 
asynchronies and cycling on the pediatric population. One of the related disadvantages is the impossibility of comparing similar effects obtained from different variables. Being that AI expresses the percentage of asynchronous events per minute and not by the cumulative time spent on each form of asynchrony. ${ }^{(6,13-15)}$

The relationship between the use of NIV NAVA and clinical outcomes were inconclusive between studies. While Vignaux et al. 2013 reported an increase in peak inspiratory pressure and mean airway pressure ${ }^{(10)}$, Chidini et al. 2016 reported divergent results on the same variables. ${ }^{11)}$ Baudin et al. 2014 and Chidini et al. 2016, showed similar results in the increase in $\mathrm{RR}^{(11,12)}$ and Vmin ${ }^{(11)}$, while Vignaux et al. 2013 did not observe significant changes in the same variables. ${ }^{(10)}$ The clinical outcomes reported by the authors were inconclusive, diverging from the findings described in the previous literature ${ }^{(4,7,8,16)}$, thus preventing confirmation of the hypothesis of correlation between favorable clinical outcomes and the use of NIV NAVA.

The use of the cross-over method adopted by the authors ${ }^{(10-13)}$, makes it impossible to correlated an intervention to specific clinical outcomes for example: length of ICU stay, total time of mechanical ventilation, risk of VILI and risk of mortality. Just as the non-standardized washout period may interfere in the analysis of the results due to the remaining therapeutic effect unknown among the interventions. Limitations related to the small size of samples with high heterogeneity among the research subjects may be associated with confounding factors in the analysis of the results, thus reducing the internal and external validity of the studies.

Part of the heterogeneity of the research subjects has a direct relationship between the age group of the population and the cause of the ARF. Younger children such as those observed in the study by Baudin et al. 2014 (12) , are more susceptible to developing ARF for viral infections, such as Acute Viral Bronchiolitis (AVB). ${ }^{(17)}$ As children with a median age older, such as those observed in the study by Ducharme et al. 2015 ${ }^{(13)}$, are more susceptible to developing ARF after surgical procedures or bacterial infections as pneumonia and SEPSIS, for example. ${ }^{(18,19)}$ Thus, it may present very heterogeneous outcomes during the treatment of ARF.

\section{CONCLUSION}

After a systematic review of the four eligible articles, we conclude that NIV NAVA has a lower AI when compared to Conventional NIV in pediatric patients with ARF. However, due to methodological limitations, clinical outcomes related to the effectiveness of gas exchange, mean ventilatory parameters, respiratory distress and use of sedatives were inconclusive.

\section{FUTURE RESEARCH DIRECTIONS:}

It is understood that new randomized clinical trials, with homogeneous samples allocated in different groups (control and intervention), using the same equipment and interfaces, as well as a longer follow-up time of participants, will be necessary to establish a more precise relationship between better synchrony and favorable clinical outcomes.

\section{REFERENCES}

1. Friedman ML, Nitu ME. Acute Respiratory Failure in Children. Pediatr Ann. 2018;47(7):e268-e73.

2. Wolfler A, Raimondi G, Pagan de Paganis C, Zoia E. The infant with severe bronchiolitis: from high flow nasal cannula to continuous positive airway pressure and mechanical ventilation. Minerva Pediatr. 2018;70(6):612-22.

3. Al Otair HA, BaHammam AS. Ventilator- and interface-related factors influencing patient-ventilator asynchrony during noninvasive ventilation. Ann Thorac Med. 2020;15(1):1-8. 
4. Suarez-Sipmann F. New modes of assisted mechanical ventilation. Medicina Intensiva (English Edition). 2014;38(4):249-60.

5. Colombo D, Cammarota G, Alemani M, Carenzo L, Barra FL, Vaschetto R, et al. Efficacy of ventilator waveforms observation in detecting patient-ventilator asynchrony. Crit Care Med. 2011;39(11):2452-7.

6. Sousa MLA, Magrans R, Hayashi FK, Blanch L, Kacmarek RM, Ferreira JC. Predictors of asynchronies during assisted ventilation and its impact on clinical outcomes: The EPISYNC cohort study. J Crit Care. 2020;57:30-5.

7. Subira C, de Haro C, Magrans R, Fernandez R, Blanch L. Minimizing Asynchronies in Mechanical Ventilation: Current and Future Trends. Respir Care. 2018;63(4):464-78.

8. Lee J, Kim HS, Jung YH, Choi CW, Jun YH. Neurally adjusted ventilatory assist for infants under prolonged ventilation. Pediatr Int. 2017;59(5):540-4.

9. JPT H, J T, J C, M C, T L, MJ P, et al. Cochrane Handbook for Systematic Reviews of Interventions version 6.0 (updated July 2019). Cochrane; 2019.

10. Vignaux L, Grazioli S, Piquilloud L, Bochaton N, Karam O, Levy-Jamet Y, et al. Patient-ventilator asynchrony during noninvasive pressure support ventilation and neurally adjusted ventilatory assist in infants and children. Pediatric critical care medicine. 2013;14(8):e357-64.

11. Chidini G, De Luca D, Conti G, Pelosi P, Nava S, Calderini E. Early Noninvasive Neurally Adjusted Ventilatory Assist Versus Noninvasive Flow-Triggered Pressure Support Ventilation in Pediatric Acute Respiratory Failure: A Physiologic Randomized Controlled Trial. Pediatr Crit Care Med. 2016;17(11):e487-e95.

12. Baudin F, Pouyau R, Cour-Andlauer F, Berthiller J, Robert D, Javouhey E. Neurally adjusted ventilator assist (NAVA) reduces asynchrony during non-invasive ventilation for severe bronchiolitis. Pediatric Pulmonology. 2015;50(12):1320-7.

13. Ducharme-Crevier L, Beck J, Essouri S, Jouvet P, Emeriaud G. Neurally adjusted ventilatory assist (NAVA) allows patient-ventilator synchrony during pediatric noninvasive ventilation: A crossover physiological study. Critical Care. 2015;19(1).

14. Thille AW, Rodriguez P, Cabello B, Lellouche F, Brochard L. Patient-ventilator asynchrony during assisted mechanical ventilation. Intensive Care Med. 2006;32(10):1515-22.

15. Chao DC, Scheinhorn DJ, Stearn-Hassenpflug M. Patient-ventilator trigger asynchrony in prolonged mechanical ventilation. Chest. 1997;112(6):1592-9.

16. Chen C, Wen T, Liao W. Neurally adjusted ventilatory assist versus pressure support ventilation in patient-ventilator interaction and clinical outcomes: a meta-analysis of clinical trials. Ann Transl Med. 2019;7(16):382.

17. Nair H, Nokes DJ, Gessner BD, Dherani M, Madhi SA, Singleton RJ, et al. Global burden of acute lower respiratory infections due to respiratory syncytial virus in young children: a systematic review and meta-analysis. Lancet. 2010;375(9725):1545-55.

18. Balcells Ramírez J, López-Herce Cid J, Modesto Alapont V. [Prevalence of mechanical ventilation in pediatric intensive care units in Spain]. An Pediatr (Barc). 2004;61(6):533-41.

19. Farias JA, Fernández A, Monteverde E, Flores JC, Baltodano A, Menchaca A, et al. Mechanical ventilation in pediatric intensive care units during the season for acute lower respiratory infection: a multicenter study. Pediatr Crit Care Med. 2012;13(2):158-64. 